\title{
Development of a multi-channel capacitive probe for electric field measurements with fine spatial and high time resolution ${ }^{\text {a) }}$
}

\author{
T. Nishizawa, ${ }^{1, b)}$ A. F. Almagri, ${ }^{1}$ W. Goodman, ${ }^{2}$ S. Ohshima, ${ }^{3}$ and J. S. Sarff ${ }^{1}$ \\ ${ }^{1)}$ Department of Physics, University of Wisconsin - Madison, Madison, Wisconsin 53706, \\ $U S A$ \\ ${ }^{2)}$ Electrical Engineering Department, University of Wisconsin - Madison, Madison, Wisconsin 53706, \\ USA \\ ${ }^{3)}$ Institute of Advanced Energy, Kyoto University, Uji, Kyoto 611-0011, Japan
}

(Dated: 12 April 2018)

A capacitive probe [Mingsheng, Tan, et al. Rev. Sci. Instrum 88, 023502 (2017)] is one of a few diagnostics that is directly sensitive to the plasma potential. Using this diagnostic technique, a Multi-channel Linear Capacitive Probe (MLCP) is developed for turbulence measurements. The MLCP has 10 spatial channels and provides 9 points of radial electric field measurements simultaneously with a spatial step of $7 \mathrm{~mm}$. A new readout circuit and a correction technique for low frequency attenuation are also developed to achieve the required spatial and time resolution. A performance test of the MLCP using a reversed field pinch plasma confirms that the MLCP resolves sub-centimeter structures of the equilibrium radial electric field profile and fluctuations up to $680 \mathrm{kHz}$.

\section{INTRODUCTION}

Measurements of electric fields provide critical information to understand transport in magnetized plasmas. For example, in the case of stellarators and heliotrons, the transport of ions and electrons depends strongly on the value of the radial electric field, $E_{r}$. The resultant radial electric field is determined by the balance of the positive and negative charge flux. In some parameter regimes, there are two solutions for $E_{r}$ (ion root and electron root) that satisfy the zero charge flux condition, and sudden changes in the sign of $E_{r}$ have been observed ${ }^{1,2}$. In the core of a Reversed Field Pinch (RFP) plasma where magnetic fields are stochastic, a positive $E_{r}$ develops in order to reduce the electron transport which is otherwise much larger than the ion transport ${ }^{3,4}$. In addition, perpendicular electric fields drive flows in a plasma through the $E \times B$ drift. Flow measurements are especially important for characterizing turbulent transport which can be suppressed by flow shear. Strong flow shear that has been observed at the location of a transport barrier $^{5,6}$ is believed to decorrelate fluctuations and improve the confinements. Also, a zonal flow with a radially localized and toroidally and poloidally symmetric structure has been identified ${ }^{7}$, and the evidence of turbulent transport regulation by the amplitude of the zonal flow has been reported $^{8}$.

Turbulence that arises from micro-instabilities has the characteristic spatial scale of ion gyro-radii or smaller. As a result, measurements of turbulence require high spatial and temporal resolution of electric field measurements. Multi-point simultaneous measurements are also necessary to study the spatiotemporal structure ${ }^{6}$. A Lang-

a) Paper published as part of the Proceedings of the 22nd Topical Conference on High-Temperature Plasma Diagnostics, San Diego, California, April, 2018.

b) nishizawa@wisc.edu muir probe $\mathrm{e}^{8-10}$ (LP) is a widely used technique to measure electric fields and corresponding $E \times B$ flows because they are relatively easy to implement, and their good sensitivity to the plasma potential, $V_{\text {plasma }}$. However, applicability of a LP is limited to cold parts of a plasma where the probe does not suffer serious damage specially when it is biased. Furthermore, a floating potential, $V_{f}$, instead of $V_{\text {plasma }}$ is often used for fast measurements because of the difficulty of achieving high time resolution and fidelity. While there are other diagnostic techniques that provide the electric fields measurements, they tend to require complex systems that are difficult to implement, such as a heavy ion beam probe, ${ }^{4,7}$.

In an effort to characterize the Trapped Electron Mode (TEM) turbulence and associated transport in the Madison Symmetric (MST) Reversed Field Pinch (RFP) through $E_{r}$ measurements, a Multi-channel Linear Capacitive Probe (MLCP) is developed. In contrast to a LP which needs a voltage sweep, or a TLP which needs biased electrodes to obtain $V_{\text {plasma }}$ from $V_{f}$, a capacitive probe is intrinsically sensitive to $V_{\text {plasma }}$ when the electron temperature is above $18 \mathrm{eV}^{11}$. Also, this probe remains operational as long as a Boron Nitride (BN) particle shield, which is known for its excellent thermal and chemical stability, survives the environment. A previously developed capacitive probe demonstrated that capacitive coupling with a BN serving as a dielectric provides the measurements of $V_{\text {plasma }}$ fluctuations associated with sawtooth crashes, which have a global spatial (MHD) structure and a characteristic frequency of below $30 \mathrm{kHz}^{11}$. Based on the expertise obtained through the previous probe development, the measurement capabilities of a capacitive probe are extended from MHD scales to micro-turbulence scales. The requirements for the MLCP are to achieve a spatial resolution of $\leq 1 \mathrm{~cm}$, which is about the ion gyro-radius in MST plasmas, to measure radially localized flows, and a time resolution of from near 0 to above $500 \mathrm{kHz}$ in the frequency domain 
to investigate multi-scale couplings.

In this paper, Sec. II discusses the design and specifications of the MLCP. The spatial resolution is quantitatively evaluated through measurements. The new readout circuit, resulting time resolution, and a new low frequency attenuation correction technique are described. Preliminary results of the $E_{r}$ measurements using the MLCP are discussed in Sec. III. Finally, a summary is given in Sec. IV

\section{MULTI-CHANNEL LINEAR CAPACITIVE PROBE}

New improvements to the concept of the capacitive probe idea have been implemented to expand the spatial and temporal capabilities. The radial size of the electrode is reduced for better radial localization of the measurement. A completely new readout and a gain correction technique are developed to extend the temporal resolution as well as the gain at lower frequency.

\section{A. Electrodes and a BN Particle shield}

As Fig. 1 (a) shows, the MLCP is equipped with 10 electrodes separated by $7 \mathrm{~mm}$. The diameter and the width of the electrodes are $11 \mathrm{~mm}$ and $2 \mathrm{~mm}$, respectively. The electrodes are covered in a BN shield with the thickness of $2 \mathrm{~mm}$ as shown in Fig. 1 (b) when inserted into a plasma. The BN shield serves as a dielectric material, and the electrodes and the plasma form capacitors. The dimensions of the electrodes and the $\mathrm{BN}$ shield are determined so that the capacitance of the plasmaelectrode couping is sufficiently large for $V_{\text {plasma }}$ to be read out. The BN shield is gradually eroded by plasmas, and replaced after having been used for several hundreds discharges.

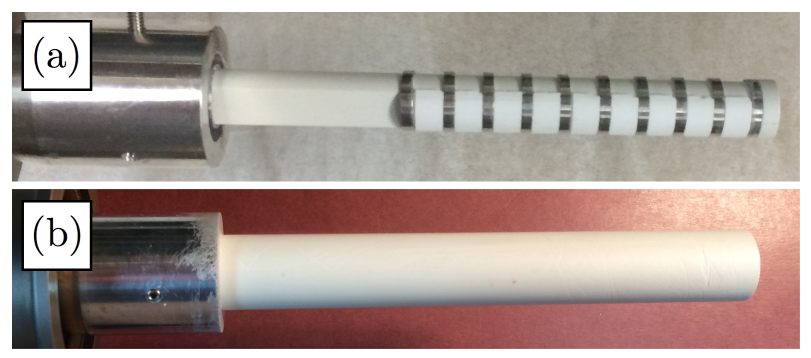

FIG. 1. A multi-channel linear capacitive probe without BN particle shield (a) and with BN particle shield (b)

\section{B. Spatial Resolution}

While the width of the electrode $(2 \mathrm{~mm})$ is small compared with the spatial separation between them $(7 \mathrm{~mm})$, the $V_{\text {plasma }}$ at the outer surface of the BN will vary along the probe axis, radial extent, which will couple to the electrodes. If coupling between an electrode and the plasma is not well-localized, and the electrode is sensitive to the plasma potential farther than $7 \mathrm{~mm}$, the measured plasma potential difference will be smoothed out. In order to confirm that the spatial resolution is not limited by the spatial extension of an electrode's sensitivity, the coupling between an electrode and the potential on the surface of the $\mathrm{BN}$ shield is measured. The BN shield is covered by an aluminum cylinder as show in Fig. 2. A function generator drives the potential of the left-half of the cylinder shown in red by a sine wave while the right-half of the cylinder shown in green is grounded. The cylinder is spatially scanned, and the output signal level of one channel is measured. The potential profile of the cylinder can be thought of as a step function. As the driven part of the cylinder moves and covers the electrode, the spatial profile of the electrode's sensitivity is integrated. The black dots in Fig. 2 (b) show the peak-peak output of one MLCP channel as the cylinder is swept through. The original electrode's sensitivity profile is assumed to a Gaussian function, and the integrated sensitivity profile is fitted using the function:

$$
\text { output }=\int_{-\infty}^{X} a_{1} \exp \left(-\frac{\left(X^{\prime}-a_{2}\right)^{2}}{a_{3}^{2}} \cdot 4 \ln 2\right) d X^{\prime}+a_{4}
$$

where $X$ is the position of the cylinder, and $a_{1}, a_{2}, a_{3}$, and $a_{4}$ are fitting parameters. the fitting function is defined so that $a_{2}$ and $a_{3}$ are the center of the electrode and the FWHM of the Gaussian sensitivity profile, respectively. Figure. 2 shows there is no serious smoothing introduced to the electric field measurements because the FWHM of the electrode's sensitivity profile is 5.6 $\mathrm{mm}$. This result shows that the MLCP can measure the electric fields at 9 points simultaneously with a spatial resolution of $7 \mathrm{~mm}$ by evaluating the plasma potential difference between adjacent electrodes.

\section{Readout Circuit}

For practical geometries, the capacitance of the plasma-electrode coupling is on the order of $\mathrm{pF}$, which leads to the output impedance on the order of $G \Omega$ at $10 \mathrm{~Hz}$, which is the characteristic frequency of MST pulse duration, and correspondingly small input currents. Therefore, the readout circuit needs to have an ultrahigh input impedance and be able to process the small currents while keeping the noise level acceptable. The internal and external connection to each electrode is about $5 \mathrm{~m}$ in length, which also poses a challenge since the capacitance of the cable $(\sim 500 \mathrm{pF})$ allows a significant portion of the input current to flow to the ground, and limits the maximum achievable input impedance of the readout circuit. Whereas a capacitive divider ${ }^{12}$ allows for a constant and reasonable high gain over a wide fre- 


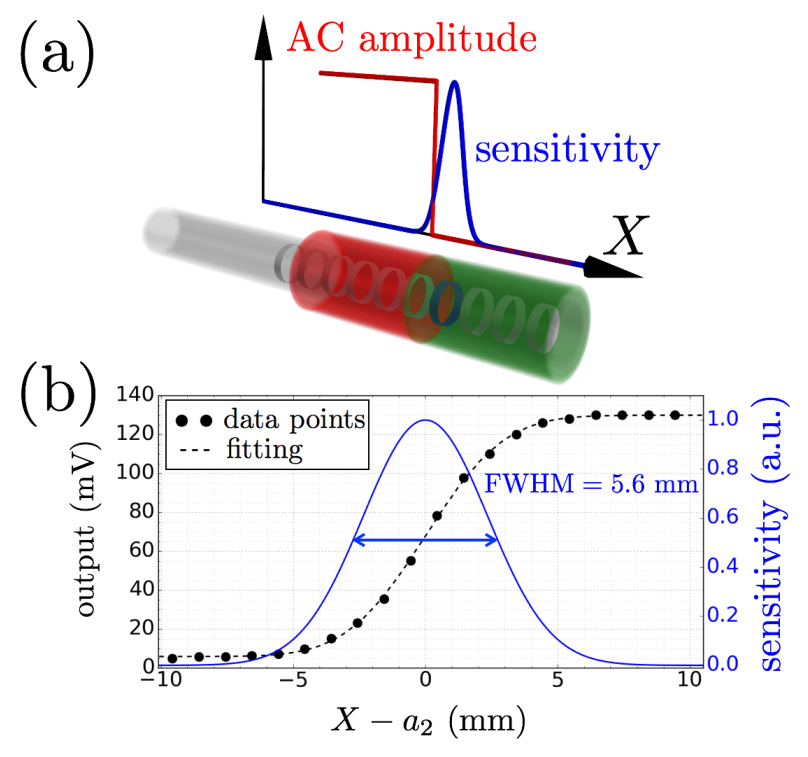

FIG. 2. (a) The MLCP and an aluminum cylinder with the red part driven and the green part grounded. (b) The integrated sensitivity of one MLCP channel.

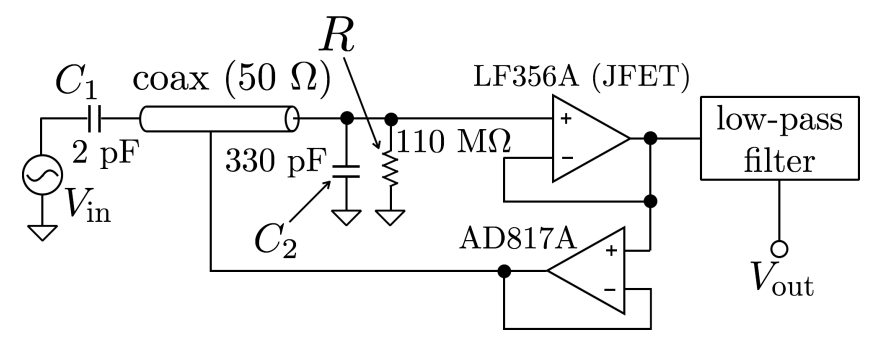

FIG. 3. The circuit diagram of the MLCP readout circuit. The capacitor between the plasma and an electrode is $C_{1}$.

quency range, there is still significant signal attenuation at low frequencies in the gains reported by $\operatorname{Refs}^{11,12}$ due to insufficient input impedance. The issues associated with a capacitive probe becomes even more challenging as the size of an electrode is reduced to attain high spatial resolution because the capacitance between a plasma and the electrode decreases, resulting in an even larger input impedance.

To simultaneously achieve fine spatial resolution with a small electrode, and a constant gain down to a low frequency, a new readout circuit shown in Fig. 3 is developed for the MLCP. This readout circuit employs "driven-guard" 13 , which drives the potential of the shield to that of the central wire so that no current flows between them. As a result, the capacitance of the coaxial cable is effectively canceled, and the use of small electrodes and longer cables becomes possible. The low-pass filter, which houses a five-pole Bessel filter, has a unity gain in the frequency range of interest and removes high frequency components above the Nyquist frequency of 1
$\mathrm{MHz}$ to prevent aliasing. The frequency characteristics of the readout circuit shown in Fig. 4 are obtained by tightly wrapping the BN shield with an aluminum foil and driving its potential by a function generator. The gain stays almost constant down to $10 \mathrm{~Hz}$. The wide bandwidth $\left(f_{3 \mathrm{~dB}}=680 \mathrm{kHz}\right)$ allows us to resolve $V_{\text {plasma }}$ or $E_{r}$ fluctuations associated with turbulence.

\section{Correction for low frequency attenuation}

The input impedance of an op-amp, LF356A (JFET) ${ }^{14}$ is $\sim 1 \mathrm{~T} \Omega$, which is much larger than $R=110 \mathrm{M} \Omega$. Therefore, a low frequency limit is given by $1 /\left[2 \pi\left(C_{1}+\right.\right.$ $\left.\left.C_{2}\right) R\right]=4.4 \mathrm{~Hz}$. While this value is a factor of three smaller than the low frequency limit reported in $\operatorname{Ref}^{11}$, MST plasmas with a duration of $\leq 100 \mathrm{~ms}$ still need corrections for low-frequency attenuation. Although this correction can be made in the frequency domain ${ }^{11}$, FFT inherently assumes periodic signals which are not necessarily appropriate, and any window function has finite frequency resolution which introduces errors.

A new correction method that is carried out in the time domain is employed for the MLCP. In Fig. $3, V_{\text {in }}$ in terms of $V_{\text {out }}$ is given by

$$
V_{\text {in }}=\frac{C_{1}+C_{2}}{C_{1}} V_{\text {out }}+\frac{1}{C_{1} R} \int V_{\text {out }} d t .
$$

The second term on the RHS corrects for low-frequency attenuation. The exact values for $C_{1}, C_{2}$, and $R$ can slightly differ from one channel to another due to the nonuniformity of the electrical components. The exact values are determined through calibration. At frequencies in the range $1 /\left[2 \pi\left(C_{1}+C_{2}\right) R\right] \ll f \ll f_{3 \mathrm{~dB}}$, the second term of the RHS in Eq. (2) is negligible, and the power gain $g$ is given by $V_{\text {out }}^{2} / V_{\mathrm{in}}^{2} \approx C_{1}^{2} /\left(C_{1}+C_{2}\right)^{2}$ which corresponds to the constant gain between $50 \mathrm{~Hz}$ and $300 \mathrm{kHz}$ in Fig. 4 . As shown in Fig. 5, when $V_{\text {in }}$ is a square pulse, $V_{\text {out }}$ decays exponentially with a time constant $\tau=\left(C_{1}+C_{2}\right) R$ after $V_{\text {in }}$ switches from one voltage level to the other. A tightly wrapped aluminum foil and a function generator are used to drive the surface potential of the BN shield, and $g$ and $\tau$ for each channel are measured. The coefficients of the RHS of Eq. (2) are given by $1 / g^{1 / 2}$ and $1 /\left(\tau g^{1 / 2}\right)$. In order to illustrate how the low frequency components are recovered, the corrected outputs of one MLCP channel for a triangle wave input are shown in Fig. 6 . The blue line is calculated by keeping only the first term on the RHS of Eq. (2), and the red line is calculated by adding the correction term for the low-frequency attenuation.By utilizing the new readout circuit and Eq. (2), both the equilibrium and fluctuating components of $V_{\text {plasma }}$ and $E_{r}$ can be measured reliably. 


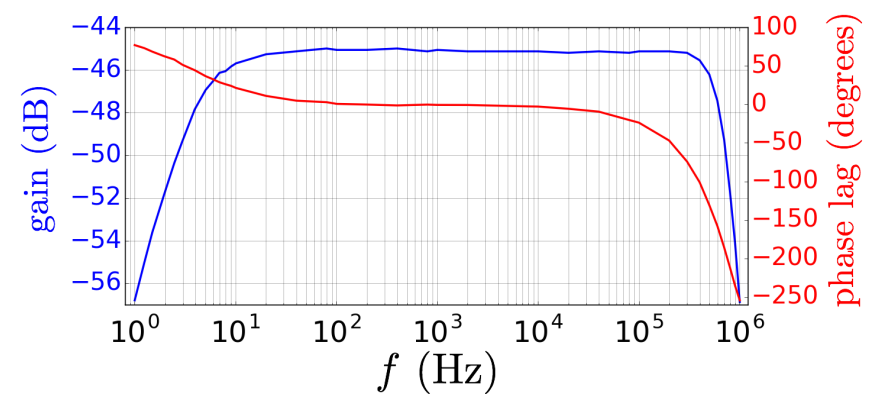

FIG. 4. The frequency characteristics of the MLCP readout circuit.

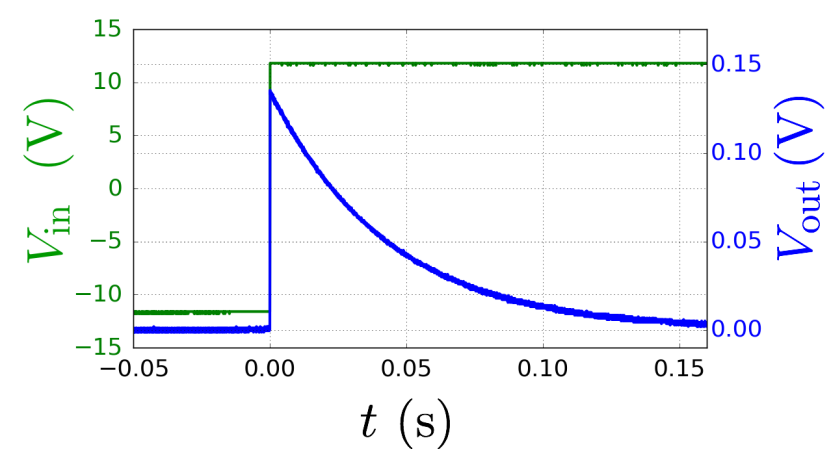

FIG. 5. A square input pulse (green) and an output of the readout circuit (blue)

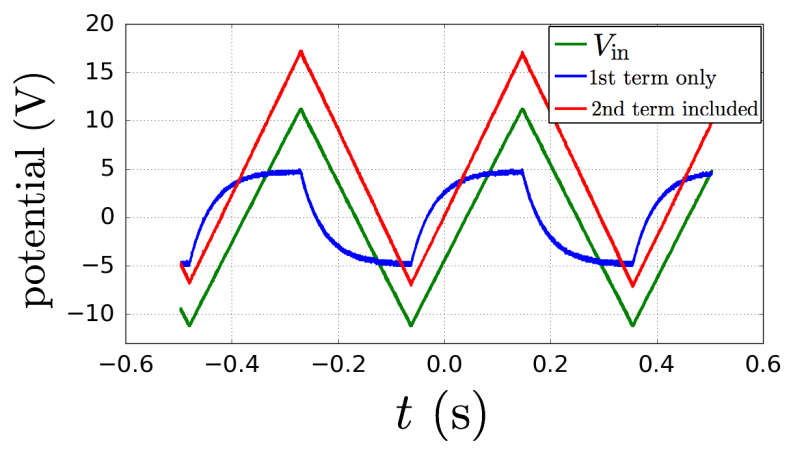

FIG. 6. A triangle input pulse (green) and output signals with a low frequency attenuation correction (red) and without a low frequency attenuation correction (blue)

\section{PRELIMINARY RESULTS}

Preliminary $E_{r}$ measurements are made by using the MLCP at MST. The edge of the MLCP is inserted by 6 $\mathrm{cm}$ from a limiter location. The time evolution of electron density and plasma current of a discharge are shown in Fig. 7 (d). A current profile control technique called Pulsed Poloidal Current Drive (PPCD) $)^{15,16}$ is applied at $10 \mathrm{~ms}$ in order to achieve an improved confinement period. Figure. 7 (a) shows the time evolution of the $E_{r}$ profile. It can be seen that the sub-centimeter structure of the $E_{r}$ profiles are resolved. In the case of the standard confinement period $\left(t<10 \mathrm{~ms}\right.$ ), the $E_{r}$ well is localized near $r-a=2 \mathrm{~cm}$. This profile is consistent with previous measurements ${ }^{17}$, indicating that the MLCP is sensitive to $V_{\text {plasma }}$ as expected. At $10 \mathrm{~ms}$ when PPCD starts, the $E_{r}$ well starts shifting inward. Around $15 \mathrm{~ms}$, the $E_{r}$ well stops moving, and a clear reduction in the magnetic field fluctuations and an increase in the soft-xray emission intensity follow as seen in Fig. 7 (c). Figure. 7 (b) shows $E_{r}$ fluctuations also decrease especially near the limiter after $15 \mathrm{~ms}$.

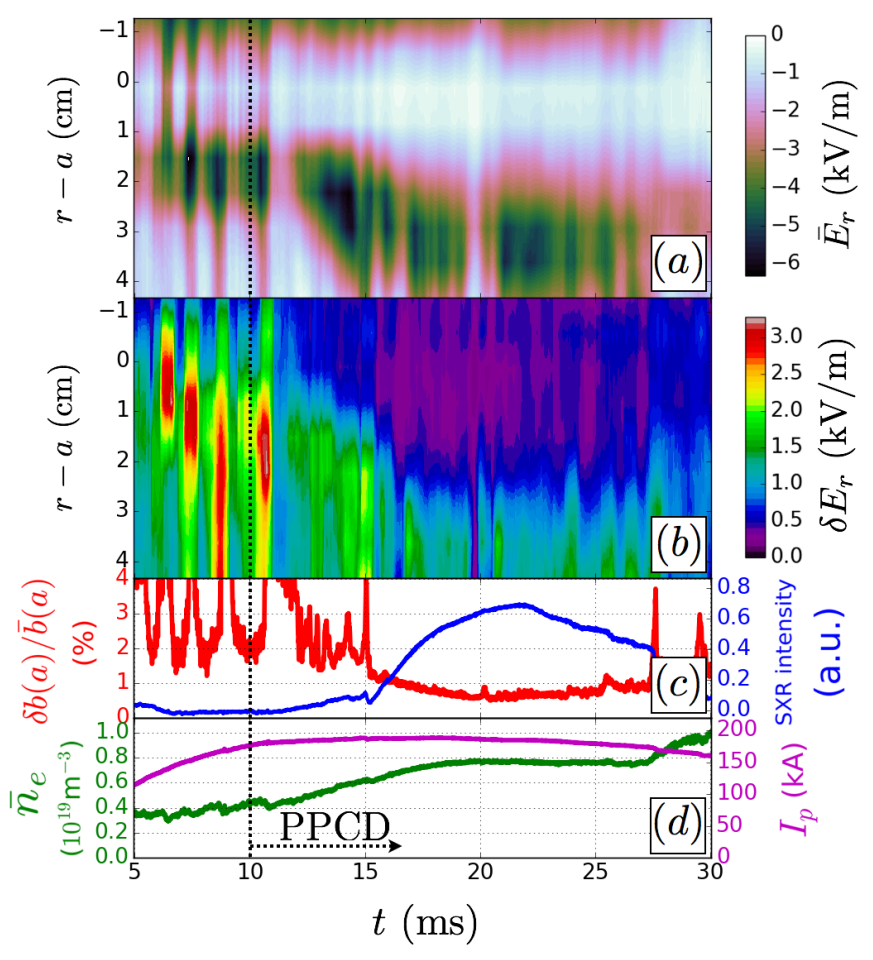

FIG. 7. Time evolution of (a) equilibrium $E_{r}$ calculated by applying a moving average filter of $500 \mu \mathrm{s}$ and (b) standard deviation of $E_{r}$ over a $500 \mu$ s time window. (c) Time evolution of magnetic fluctuation amplitude on the wall and soft-xray emision intensity. (d) Time evolution of the line-averaged electron density and plasma current. $a=50 \mathrm{~cm}$. PPCD starts at $10 \mathrm{~ms}$.

The $E_{r}$ power spectral densities for with and without PPCD at $r / a=0.91$ are shown in Fig. 8. Before the onset of PPCD, most of the power is concentrated below 30 $\mathrm{kHz}$ which is the characteristic frequency range of tearing modes ${ }^{18}$. During the PPCD-induced improved confinement period, the $E_{r}$ fluctuations are reduced over all frequencies except near $50 \mathrm{kHz}$. This feature is thought to be related to the electron density fluctuations that peak near $50 \mathrm{kHz}$ observed by using far-infrared interferometry $(\mathrm{FIR})^{19}$, and trapped-electron modes are responsible for it as was shown in gyro-kinetic simulations ${ }^{20}$. For both time periods, the measured power spectral density 
of the $E_{r}$ fluctuations are well above the noise level up to $680 \mathrm{kHz}$ which is the $3 \mathrm{~dB}$ point of the readout circuit.

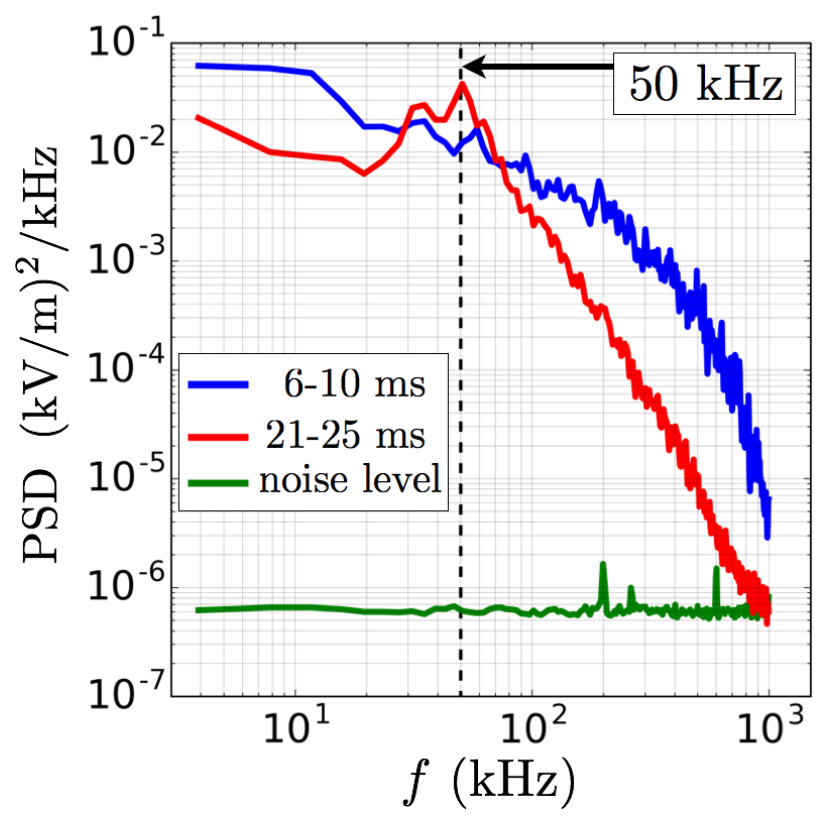

FIG. 8. The power spectral density spectra of the $E_{r}$ fluctuation measured at $\mathrm{r} / \mathrm{a}=0.91(r-a=4.4 \mathrm{~cm})$ for standard (6-10 ms) and improved confinement periods $(21-25 \mathrm{~ms})$.

\section{SUMMARY}

In order to characterize turbulence and associated transport in a RFP plasma, the MCLP probe has been developed to provide the $E_{r}$ measurements at 9 points with a separation of $7 \mathrm{~mm}$ simultaneously. The high time resolution has been made possible through the new readout circuit with an extremely high input impedance and a driven-guard. The new technique to correct low frequency attenuation has also been developed to calculate the equilibrium $V_{\text {plasma }}$ and $E_{r}$. Th preliminary measurements have been conducted, and it is demonstrated that the MLCP reliably provides the $E_{r}$ measurements with a high signal-to-noise ratio and with improved time and spatial resolution.

\section{SUPPLEMENTARY MATERIAL}

See supplementary material for the digital format of the data shown in this paper.

\section{ACKNOWLEDGMENTS}

This work is supported by the U.S. Department of Energy, Office of Science, Office of Fusion Energy Sciences under Award Number DE-FC02-05ER54814, and NINS (National Institutes of Natural Sciences)/NIFS (National Institute for Fusion Science) under the project, "Strategic International Research Interaction Acceleration Initiative".

${ }^{1}$ A. Fujisawa, H. Iguchi, T. Minami, Y. Yoshimura, K. Tanaka, K. Itoh, H. Sanuki, S. Lee, M. Kojima, S.-I. Itoh, M. Yokoyama, S. Kado, S. Okamura, R. Akiyama, K. Ida, M. Isobe, S. Nishimura, M. Osakabe, I. Nomura, A. Shimizu, C. Takahashi, K. Toi, K. Matsuoka, Y. Hamada, and M. Fujiwara, Physics of Plasmas 7, 4152 (2000), https://aip.scitation.org/doi/pdf/10.1063/1.1290483.

${ }^{2}$ K. Ida, H. Funaba, S. Kado, K. Narihara, K. Tanaka, Y. Takeiri, Y. Nakamura, N. Ohyabu, K. Yamazaki, M. Yokoyama, S. Murakami, N. Ashikawa, P. C. deVries, M. Emoto, M. Goto, H. Idei, K. Ikeda, S. Inagaki, N. Inoue, M. Isobe, K. Itoh, O. Kaneko, K. Kawahata, K. Khlopenkov, A. Komori, S. Kubo, R. Kumazawa, Y. Liang, S. Masuzaki, T. Minami, J. Miyazawa, T. Morisaki, S. Morita, T. Mutoh, S. Muto, Y. Nagayama, H. Nakanishi, K. Nishimura, N. Noda, T. Notake, T. Kobuchi, S. Ohdachi, K. Ohkubo, Y. Oka, M. Osakabe, T. Ozaki, R. O. Pavlichenko, B. J. Peterson, A. Sagara, K. Saito, S. Sakakibara, R. Sakamoto, H. Sanuki, H. Sasao, M. Sasao, K. Sato, M. Sato, T. Seki, T. Shimozuma, M. Shoji, H. Suzuki, S. Sudo, N. Tamura, K. Toi, T. Tokuzawa, Y. Torii, K. Tsumori, T. Yamamoto, H. Yamada, I. Yamada, S. Yamaguchi, S. Yamamoto, Y. Yoshimura, K. Y. Watanabe, T. Watari, Y. Hamada, O. Motojima, and M. Fujiwara, Phys. Rev. Lett. 86, 5297 (2001).

${ }^{3}$ H. Ji, H. Toyama, K. Miyamoto, S. Shinohara, and A. Fujisawa, Phys. Rev. Lett. 67, 62 (1991).

${ }^{4}$ D. R. Demers, X. Chen, P. M. Schoch, and P. J. Fimognari, Review of Scientific Instruments 81, 10E109 (2010), https://doi.org/10.1063/1.3479109.

${ }^{5}$ E. J. Synakowski, Plasma Physics and Controlled Fusion 40, 581 (1998).

${ }^{6}$ T. Estrada, C. Hidalgo, T. Happel, and P. H. Diamond, Phys. Rev. Lett. 107, 245004 (2011).

${ }^{7}$ A. Fujisawa, K. Itoh, H. Iguchi, K. Matsuoka, S. Okamura, A. Shimizu, T. Minami, Y. Yoshimura, K. Nagaoka, C. Takahashi, M. Kojima, H. Nakano, S. Ohsima, S. Nishimura, M. Isobe, C. Suzuki, T. Akiyama, K. Ida, K. Toi, S.-I. Itoh, and P. H. Diamond, Phys. Rev. Lett. 93, 165002 (2004).

${ }^{8}$ G. Birkenmeier, M. Ramisch, B. Schmid, and U. Stroth, Phys. Rev. Lett. 110, 145004 (2013).

${ }^{9}$ Y. Nagashima, K. Hoshino, A. Ejiri, K. Shinohara, Y. Takase, K. Tsuzuki, K. Uehara, H. Kawashima, H. Ogawa, T. Ido, Y. Kusama, and Y. Miura, Phys. Rev. Lett. 95, 095002 (2005).

${ }^{10}$ K. J. Zhao, T. Lan, J. Q. Dong, L. W. Yan, W. Y. Hong, C. X. Yu, A. D. Liu, J. Qian, J. Cheng, D. L. Yu, Q. W. Yang, X. T. Ding, Y. Liu, and C. H. Pan, Phys. Rev. Lett. 96, 255004 (2006).

${ }^{11}$ M. Tan, D. R. Stone, J. C. Triana, A. F. Almagri, G. Fiksel, W. Ding, J. S. Sarff, K. J. McCollam, H. Li, and W. Liu, Review of Scientific Instruments 88, 023502 (2017), https://doi.org/10.1063/1.4975095.

${ }^{12}$ J. A. Schmidt, Review of Scientific Instruments 39, 1297 (1968), https://doi.org/10.1063/1.1683655.

${ }^{13}$ A. Rich, Analog Dialogue 17, 8 (1983).

${ }^{14}$ Texas Instruments, "LFx5xJFET Input Operational Amplifiers," http://www.ti.com/lit/ds/symlink/lf356.pdf (2015).

${ }^{15}$ B. E. Chapman, A. F. Almagri, J. K. Anderson, T. M. Biewer, P. K. Chattopadhyay, C.-S. Chiang, D. Craig, D. J. Den Hartog, G. Fiksel, C. B. Forest, A. K. Hansen, D. Holly, N. E. Lanier, R. O'Connell, S. C. Prager, J. C. Reardon, J. S. Sarff, M. D. Wyman, D. L. Brower, W. X. Ding, Y. Jiang, S. D. Terry, P. Franz, L. Marrelli, and P. Martin, Physics of Plasmas 9, 2061 (2002), https://doi.org/10.1063/1.1456930.

${ }^{16}$ J. K. Anderson, J. Adney, A. Almagri, A. Blair, D. L. Brower, M. Cengher, B. E. Chapman, S. Choi, D. Craig, D. R. Demers, D. J. Den Hartog, B. Deng, W. X. Ding, F. Ebrahimi, D. En- 
nis, G. Fiksel, C. B. Forest, P. Franz, J. Goetz, R. W. Harvey, D. Holly, B. Hudson, M. Kaufman, T. Lovell, L. Marrelli, P. Martin, K. McCollam, V. V. Mirnov, P. Nonn, R. O'Connell, S. Oliva, P. Piovesan, S. C. Prager, I. Predebon, J. S. Sarff, G. Spizzo, V. Svidzinski, M. Thomas, and M. D. Wyman, Physics of Plasmas 12, 056118 (2005), https://doi.org/10.1063/1.1883666.

${ }^{17}$ V. Antoni, D. Desideri, E. Martines, G. Serianni, and L. Tramontin, Phys. Rev. Lett. 79, 4814 (1997).
${ }^{18}$ W. X. Ding, D. L. Brower, D. Craig, B. E. Chapman, D. Ennis, G. Fiksel, S. Gangadhara, D. J. Den Hartog, V. V. Mirnov, S. C. Prager, J. S. Sarff, V. Svidzinski, P. W. Terry, and T. Yates, Physics of Plasmas 15, 055901 (2008), https://doi.org/10.1063/1.2837047.

${ }^{19}$ J. R. Duff, Z. R. Williams, D. L. Brower, B. E. Chapman, W. X. Ding, M. J. Pueschel, J. S. Sarff, and P. W. Terry, Physics of Plasmas 25, 010701 (2018), https://doi.org/10.1063/1.5010198.

${ }^{20}$ Z. R. Williams, M. J. Pueschel, P. W. Terry, and T. Hauff, Physics of Plasmas 24, 122309 (2017), https://doi.org/10.1063/1.5000252. 